\title{
ON HILBERT-SCHMIDT COMPATIBILITY
}

\author{
Denis Potapov, Anna Skripka And Fedor SukocheV
}

\begin{abstract}
Guided by important examples of differential operators, we obtain sufficient conditions for Hilbert-Schmidt compatibility of operators and apply these conditions in spectral perturbation theory.
\end{abstract}

Mathematics subject classification (2010): 47A55, 47B10.

Keywords and phrases: Double operator integral, spectral shift function, Hilbert-Schmidt compatibility.

\section{REFERENCES}

[1] N. A. Azamov, F. A. Sukochev, Spectral averaging for trace compatible operators, Proc. AMS 136, 5 (2008), 1769-1778.

[2] N. A. Azamov, A. L. Carey, P. G. Dodds, F. A. Sukochev, Operator integrals, spectral shift, and spectral flow, Canad. J. Math. 61, 2 (2009), 241-263.

[3] N. A. Azamov, A. L. Carey, F. A. Sukochev, The spectral shift function and spectral flow, Comm. Math. Phys. 276, 1 (2007), 51-91.

[4] M. Sh. Birman, M. SolomyaK, Double operator integrals in a Hilbert space, Integral Equations Operator Theory 47, 2 (2003), 131-168.

[5] J.-M. Bouclet, Traces formulae for relatively Hilbert-Schmidt perturbations, Asymptot. Anal. 32, 3-4 (2002), 257-291.

[6] A. Connes And H. Moscovici, The local index formula in noncommutative geometry, Geom. Funct. Anal. 5, 2 (1995), 174-243.

[7] V. Gayral, J. M. Gracia-Bondía, B. Iochum, T. Schücker and J. C. VÁrilly, Moyal planes are spectral triples, Commun. Math. Phys. 246 (2004), 569-623.

[8] K. DyKemA, A. SkRIPKA, Higher order spectral shift, J. Funct. Anal. 257 (2009), 1092-1132.

[9] L. S. KoplienKo, Trace formula for perturbations of nonnuclear type, Sibirsk. Mat. Zh. 25 (1984), 62-71 (Russian). Translation: Siberian Math. J. 25 (1984), 735-743.

[10] M. G. KREIN, On a trace formula in perturbation theory, Matem. Sbornik 33 (1953), 597-626 (Russian).

[11] B. De Pagter, F. A. Sukochev, H. Witvliet, Double operator integrals, J. Funct. Anal. 192, 1 (2002), 52-111.

[12] V. V. PELLER, Hankel operators in the theory of perturbations of unitary and self-adjoint operators, Funktsional. Anal. i Prilozhen. 19, 2 (1985), 37-51.

[13] V. V. PELLER, Hankel operators in the perturbation theory of unbounded self-adjoint operators. Analysis and partial differential equations, Lecture Notes in Pure and Applied Mathematics, 122, Dekker, New York, 1990, pp. 529-544.

[14] V. V. Peller, An extension of the Koplienko-Neidhardt trace formulae, J. Funct. Anal. 221 (2005), 456-481.

[15] V. V. PELLER, Multiple operator integrals and higher operator derivatives, J. Funct. Anal. 223 (2006), 515-544.

[16] G. PIsIER AND Q. XU, Non-commutative $L^{p}$-spaces, Handbook of the geometry of Banach spaces, Vol. 2, North-Holland, Amsterdam, 2003, pp. 1459-1517.

[17] D. Potapov and F. Sukochev, Double operator integrals and submajorization, Math. Modl. Natr. Ph. 5, 4 (2010), 317-339. 
[18] D. Potapov AND F. Sukochev, Operator-Lipschitz functions in Schatten-von Neumann classes, Acta Math., to appear, arXiv:0904.4095.

[19] D. Potapov, A. Skripka, F. SuKochev, Spectral shift function of higher order, preprint, arXiv:0912.3056.

[20] D. Potapov And F. Sukochev, Unbounded Fredholm modules and double operator integrals, J. reine. angew. Math. 626 (2009), 159-185.

[21] M. ReED AND B. Simon, Methods of modern mathematical physics. I, second ed., Academic Press Inc. [Harcourt Brace Jovanovich Publishers], New York, 1980.

[22] J. T. SchwARTZ, Nonlinear functional analysis, Gordon and Breach Science Publishers, New YorkLondon-Paris, 1969.

[23] B. Simon, Trace ideals and their applications, Second Edition, Mathematical Surveys and Monographs, 120. AMS, Providence, RI, 2005.

[24] A. SKRIPKA, Multiple operator integrals and spectral shift, Illinois J. Math., to appear, arXiv:0907.0432.

[25] A. SKRIPKA, Trace inequalities and spectral shift, Oper. Matrices, 3, 2 (2009), 241-260.

[26] M. TAKESAKI, Theory of operator algebras. II, Encyclopaedia of Mathematical Sciences, vol. 125, Springer-Verlag, Berlin, 2003.

[27] M. TERP, $L^{p}$-spaces associated with von Neumann algebras, Copenhagen University, 1981. 